PROCEEDINGS OF THE

AMERICAN MATHEMATICAL SOCIETY

Volume 139, Number 3, March 2011, Pages 1127-1136

S 0002-9939(2010)10674-3

Article electronically published on October 27, 2010

\title{
PRESCRIBING THE SYMMETRIC FUNCTION OF THE EIGENVALUES OF THE SCHOUTEN TENSOR
}

\author{
YAN HE AND WEIMIN SHENG
}

(Communicated by Matthew J. Gursky)

\begin{abstract}
In this paper we study the problem of conformally deforming a metric to a prescribed symmetric function of the eigenvalues of the Schouten tensor on compact Riemannian manifolds with boundary. We prove its solvability and the compactness of the solution set, provided the Ricci tensor is nonnegative-definite.
\end{abstract}

\section{INTRODUCTION}

Let $\left(M^{n}, g\right)$ be a smooth, compact Riemannian manifold with totally geodesic boundary of dimension $n \geq 3$. The Schouten tensor of $g$ is defined by

$$
A_{g}=\frac{1}{n-2}\left(\operatorname{Ric}_{g}-\frac{R_{g}}{2(n-1)} g\right),
$$

where Ric and $R$ are the Ricci and scalar curvatures of $g$, respectively.

Let $\sigma_{k}: R^{n} \rightarrow R$ be the $k$-th elementary symmetric function $(1 \leq k \leq n)$

$$
\sigma_{k}(x)=\sum_{1 \leq i_{1}<\cdots<i_{k} \leq n} x_{i_{1}} \cdots x_{i_{k}},
$$

$\Gamma_{k}$ the corresponding open, convex cone, i.e. $\Gamma_{k}=\left\{x \in R^{n} \mid \sum_{i}(x)>0,1 \leq i \leq k\right\}$. Let

$$
\Sigma_{\theta}=\left\{x=\left(x_{1}, \cdots, x_{n}\right) \in R^{n} \mid \quad \min x_{i}+\theta \sum x_{i}>0\right\} .
$$

Now let us consider the general symmetric function $F$ defined on $\Gamma\left(\Gamma_{n} \subset \Gamma \subset\right.$ $\left.\Sigma_{\frac{1}{n-2}}\right)$ and satisfying

$\left(C_{1}\right) F$ is positive and $F=0$ on $\partial \Gamma$;

$\left(C_{2}\right) F$ is concave;

$\left(C_{3}\right) F$ is invariant under exchange of variables;

$\left(C_{4}\right) F$ is homogeneous of degree 1;

$\left(C_{5}\right) \lim _{s \rightarrow \infty} F(s x)=\infty, \quad \forall x \in \Gamma$

$\left(C_{6}\right) F(x) \leq \varrho \sigma_{1}(x)$ in $\Gamma$ and $F(1, \cdots, 1)=n \varrho$, where $\varrho$ is a positive constant;

$\left(C_{7}\right) \frac{\partial F}{\partial x_{i}} \geq \Sigma \frac{F}{\sigma_{1}}$ for some constant $\Sigma>0$ for all $i$.

Received by the editors January 31, 2010.

2010 Mathematics Subject Classification. Primary 53C21; Secondary 35J60.

Key words and phrases. Conformal geometry, prescribing curvature, Ricci tensor.

This work was partially supported by NSFC Grants 10771189 and 10831008.

(C)2010 American Mathematical Society 
We need $\left(C_{1}\right)-\left(C_{4}\right)$ to ensure that the elliptic equations are solvable. If $F$ additionally satisfies $\left(C_{5}\right)$, then the Liouville Theorem in [12] is applicable. Condition $\left(C_{6}\right)$ says that the Newton-Maclaurin inequality with respect to the function $F$ holds. $F=\sigma_{k}^{1 / k}$ satisfies condition $\left(C_{7}\right)$.

We denote $[g]=\left\{\tilde{g} \mid \tilde{g}=e^{-2 u} g\right\}$. We call the metric $\hat{g}=e^{-2 u} g$ (as well as the function $u) \Gamma$-admissible, or simply admissible, if $\hat{g} \in\left\{\tilde{g} \in[g] \mid \lambda\left(\tilde{g}^{-1} A_{\tilde{g}}\right) \in \Gamma\right\}$. Here, $\lambda\left(\tilde{g}^{-1} A_{\tilde{g}}\right)=\left(\lambda_{1}, \cdots, \lambda_{n}\right)$ denote the eigenvalues of $\tilde{g}^{-1} A_{\tilde{g}}$.

In this paper we study the existence of some prescribing problems and the compactness of the solution set. The main result is as follows.

Theorem 1.1. Let $\left(M^{n}, g\right)$ be a compact $n$-dimensional Riemannian manifold with totally geodesic boundary. Let $F$ be a symmetric function satisfying $\left(C_{1}\right)-\left(C_{7}\right)$ on $\Gamma$ with $\Gamma_{n} \subset \Gamma \subset \Sigma_{\frac{1}{n-2}}$. If the manifold $(M, g)$ is not conformally equivalent to a hemisphere, then for any positive function $f$, there exists an admissible conformal metric $\tilde{g}=e^{-2 u} g$ with totally geodesic boundary satisfying

$$
F\left(\lambda\left(\tilde{g}^{-1} A_{\tilde{g}}\right)\right)=f .
$$

Additionally, the set of all such solutions is compact in the $C^{m}$-topology for any $m \geq 0$.

We can get the following corollary from Theorem 1.1 immediately. That is, to find a conformal metric $\tilde{g}$ with nonnegative $\operatorname{Ric}_{\tilde{g}}$ such that

$$
\operatorname{det}\left(\mu\left(\tilde{g}^{-1} \operatorname{Ric}_{\tilde{g}}\right)\right)=f^{n},
$$

where $\mu\left(\tilde{g}^{-1} \operatorname{Ric}_{\tilde{g}}\right)=\left(\mu_{1}, \cdots, \mu_{n}\right)$ are the eigenvalues of $\tilde{g}^{-1} \operatorname{Ric}_{\tilde{g}}$ and $f(x)$ is a positive function.

Since $\operatorname{Ric}_{\tilde{g}}=(n-2) A_{\tilde{g}}+\sigma_{1}\left(\lambda\left(\tilde{g},{ }^{-1} A_{\tilde{g}}\right)\right) \tilde{g}$ if we define $F(\lambda)=\sigma_{n}^{1 / n}((n-2) \lambda$ $\left.+\left(\sum_{i=1}^{n} \lambda_{i}\right)\right)$ and $\Gamma=\{\lambda \mid F(\lambda)>0\}$, then

$$
\begin{aligned}
& \operatorname{det}^{1 / n}\left(\mu\left(\tilde{g}^{-1} \operatorname{Ric}_{\tilde{g}}\right)\right) \\
= & \sigma_{n}^{1 / n}\left(\mu\left(g^{-1}\left[(n-2)\left(d u \otimes d u-|\nabla u|^{2} g\right)+(n-2) \nabla^{2} u+\Delta u g+\operatorname{Ric}_{g}\right]\right)\right) \\
= & F\left(\lambda\left(g^{-1}\left[\nabla^{2} u+d u \otimes d u-\frac{1}{2}|\nabla u|^{2} g+A_{g}\right]\right)\right),
\end{aligned}
$$

where $\mu=(n-2) \lambda+\sum_{i=1}^{n} \lambda_{i}$. From the definition, it is easy to verify that $F$ satisfies $\left(C_{1}\right)-\left(C_{5}\right)$, since

$$
\frac{\partial F}{\partial \lambda_{i}}=\frac{\partial\left(\sigma_{n}^{1 / n}\right)}{\partial \mu_{s}}\left(1+(n-2) \delta_{i}^{s}\right)
$$

and

$$
\frac{\partial^{2} F}{\partial \lambda_{i} \partial \lambda_{j}}=\left(1+(n-2) \delta_{i}^{s}\right) \frac{\partial^{2}\left(\sigma_{n}^{1 / n}\right)}{\partial \mu_{s} \partial \mu_{t}}\left(1+(n-2) \delta_{j}^{t}\right) .
$$

Moreover, from

$$
\begin{aligned}
F\left(\lambda\left(\tilde{g}^{-1} A_{\tilde{g}}\right)\right) & =\sigma_{n}^{1 / n}\left(\mu\left(\tilde{g}^{-1} \operatorname{Ric}_{\tilde{g}}\right)\right) \\
& \leq \frac{1}{n} \sigma_{1}\left(\mu\left(\tilde{g}^{-1} \operatorname{Ric}_{\tilde{g}}\right)\right) \\
& =\frac{2 n-2}{n} \sigma_{1}\left(\lambda\left(\tilde{g}^{-1} A_{\tilde{g}}\right)\right)
\end{aligned}
$$


we know that $F$ satisfies $\left(C_{6}\right)$ with $\varrho=\frac{2 n-2}{n}$. Thus (1.1) turns out to be a proper equation with respect to the Schouten tensor. Furthermore, as in [6] and [15], by use of the volume comparison theorem, the $C^{0}$ estimate of the solutions of such an equation can be derived if Ric $\geq 0$. In other words, the condition $\Gamma \subset \Sigma_{\frac{1}{n-2}}$ ensures that the volume comparison theorem is applicable, where the eigenvalues $\lambda$ of the Schouten tensor satisfy $(n-2) \lambda+\sum_{i=1}^{n} \lambda_{i} \geq 0$ if and only if the eigenvalues of the Ricci tensor $\mu \geq 0$. Similarly, on the manifold with totally geodesic boundary, based on the boundary $C^{1}, C^{2}$ estimates with the Neumann boundary condition for the general symmetric function ([2] or [9], etc.), we can get

Corollary 1.2. Let $(M, g)$ be a compact $n$-dimensional Riemannian manifold with totally geodesic boundary and with the Ricci tensor semi-positive-definite. If it is not conformally equivalent to a hemisphere, then for any positive function $f$, there exists a conformal metric $\tilde{g}=e^{-2 u} g$ with totally geodesic boundary and $\operatorname{Ric}_{\tilde{g}} \geq 0$ and

$$
\operatorname{det}\left(\mu\left(\tilde{g}^{-1} \operatorname{Ric}_{\tilde{g}}\right)\right)=f^{n} .
$$

Additionally, the set of all such solutions is compact in the $C^{m}$-topology for any $m \geq 0$.

Remark 1.3. The conformal problem with respect to the Ricci tensor has been studied extensively. In [13] and [8], the authors studied the negative Ricci curvature and proved that there exists a conformal metric $\tilde{g}$ with negative Ricci tensor $\operatorname{Ric}_{\tilde{g}}$ such that

$$
\operatorname{det}\left(\mu\left(\tilde{g}^{-1} \operatorname{Ric}_{\tilde{g}}\right)\right)=\text { const } .
$$

When the Ricci tensor is positive-definite, in [5], Guan and Wang derived a conformal metric with a constant smallest eigenvalue of the Ricci tensor. In [15], Trudinger and Wang proved the prescribing problem of a positive Ricci tensor on a closed manifold.

This paper is organized as follows. We begin with some preliminaries in Section 2. In Section 3, we will discuss the deformation and a priori estimates. The proof of Theorem 1.1 is in Section 4.

\section{Preliminaries}

We first introduce Fermi coordinates in a boundary neighborhood. In these local coordinates, we take the geodesic in the inner normal direction $\nu=\frac{\partial}{\partial x^{n}}$ parameterized by arc length, and $\left(x^{1}, \ldots, x^{n-1}\right)$ forms a local chart on the boundary where $x^{n}=0$. The metric can be expressed as

$$
g=g_{\alpha \beta} d x^{\alpha} d x^{\beta}+\left(d x^{n}\right)^{2} .
$$

The Greek letters $\alpha, \beta, \gamma, \ldots$ stand for the tangential direction indices, $1 \leq \alpha, \beta, \gamma, \ldots$ $\leq n-1$, while the Latin letters $i, j, k, \ldots$ stand for the full indices, $1 \leq i, j, k, \ldots \leq n$ (see [4] and [1]).

We denote the functions, tensors and covariant differentiations with respect to the induced metric on the boundary by a bar (e.g. $\left.\bar{\Gamma}_{\beta \gamma}^{\alpha}, \bar{R}_{\alpha \beta}\right)$. Then the Christoffel symbols on the boundary satisfy

$$
\bar{\Gamma}_{\alpha \beta}^{\gamma}=\frac{1}{2} g^{\gamma \delta}\left(\frac{\partial g_{\alpha \delta}}{\partial x^{\beta}}+\frac{\partial g_{\beta \delta}}{\partial x^{\alpha}}-\frac{\partial g_{\alpha \beta}}{\partial x^{\delta}}\right)=\Gamma_{\alpha \beta}^{\gamma}
$$

and $\Gamma_{n n}^{n}=0, \Gamma_{n n}^{\alpha}=0, \Gamma_{n \alpha}^{n}=0$. 
Let us denote $\frac{\partial}{\partial x^{i}}$ by $\partial_{i}$. The boundary is called umbilic if the second fundamental form $L_{\alpha \beta}=\tau g_{\alpha \beta}$, where $\tau$ is a function defined on $\partial M$. Since the boundary $\partial M$ is connected, by the Schur Theorem, $\tau=$ const. A totally geodesic boundary is umbilic with $\tau=0$.

Thus $\left.\Gamma_{\alpha \beta}^{n}\right|_{\partial M}=L_{\alpha \beta}=\tau g_{\alpha \beta}$ and $\left.\Gamma_{n \beta}^{\alpha}\right|_{\partial M}=-L_{\alpha \gamma} g^{\gamma \beta}=-\tau \delta_{\alpha}^{\beta}$.

Under the conformal metric $\tilde{g}=e^{-2 u} g$, the functions, tensors and the covariant differentiations with respect to $\tilde{g}$ are denoted by a tilde (e.g. $\left.A_{\tilde{g}}, \tilde{L}_{\alpha \beta}\right)$.

Let $[g]$ be the set of metrics conformal to $g$. For $\tilde{g}=e^{-2 u} g \in[g]$, we consider the equation

$$
F\left(\lambda\left(\tilde{g}^{-1} A_{\tilde{g}}\right)\right)=f .
$$

The Schouten tensor transforms according to the formula

$$
A_{\tilde{g}}=\nabla^{2} u+d u \otimes d u-\frac{1}{2}|\nabla u|^{2} g+A_{g}
$$

where $\nabla u$ and $\nabla^{2} u$ denote the gradient and Hessian of $u$ with respect to $g$. Consequently, (2.1) is equivalent to

$$
F\left(\lambda\left(g^{-1}\left[\nabla^{2} u+d u \otimes d u-\frac{1}{2}|\nabla u|^{2} g+A_{g}\right]\right)\right)=f(x) e^{-2 u} .
$$

Then the second fundamental form satisfies

$$
\tilde{L}_{\alpha \beta} e^{u}=\frac{\partial u}{\partial \nu} g_{\alpha \beta}+L_{\alpha \beta} .
$$

Note that the umbilicity is conformally invariant. When the boundary is umbilic, the above formula becomes

$$
\tilde{\tau} e^{-u}=\frac{\partial u}{\partial \nu}+\tau
$$

where $\tilde{L}_{\alpha \beta}=\tilde{\tau} \tilde{g}_{\alpha \beta}$.

Therefore, whereas the initial metric $g$ on the manifold $M$ is with totally geodesic boundary $\partial M$, the boundary of the manifold $M$ with conformal metric $\tilde{g}=e^{-2 u} g$ is still totally geodesic if and only if $\frac{\partial u}{\partial \nu}=0$.

Therefore, in order to prove Theorem 1.1, we need to find admissible solutions of the following equation:

$$
\left\{\begin{array}{lr}
F\left(\lambda\left(g^{-1}\left[\nabla^{2} u+d u \otimes d u-\frac{1}{2}|\nabla u|^{2} g+A_{g}\right]\right)\right)=f(x) e^{-2 u} & \text { in } M \\
\frac{\partial u}{\partial \nu}=0 & \text { on } \partial M .
\end{array}\right.
$$

\section{Deformation, $C^{1}$ and $C^{2}$ estimates}

To prove the existence of a solution to the equation (2.2), we employ the following deformation, which is defined in [7]:

$$
\left\{\begin{array}{lr}
F\left(\lambda\left(g^{-1}\left[\varsigma(1-\psi(t)) g+\psi(t) A_{g}+\nabla^{2} u+d u \otimes d u-\frac{1}{2}|\nabla u|^{2} g\right]\right)\right) & \\
=\psi(t) f(x) e^{-2 u}+(1-t)\left(\int e^{-(n+1) u}\right)^{2 / n+1} & \text { in } M \\
\frac{\partial u}{\partial \nu}=0 & \text { on } \partial M
\end{array}\right.
$$

where $\psi \in C^{1}[0,1]$ satisfies $0 \leq \psi(t) \leq 1, \psi(0)=0, \psi(t)=1$ for $t \geq \frac{1}{2}$, and $\varsigma=(n \varrho)^{-1} \operatorname{vol}\left(M_{g}\right)^{\frac{2}{n+1}}$, where $F(1, \cdots, 1)=n \varrho$. 
Similarly to [7, at $t=1$, (3.1) becomes (2.2), while at $t=0$, it becomes

$$
\left\{\begin{array}{lr}
F\left(\lambda\left(g^{-1}\left[\varsigma g+\nabla^{2} u+d u \otimes d u-\frac{1}{2}|\nabla u|^{2} g\right]\right)\right)=\left(\int e^{-(n+1) u}\right)^{\frac{2}{n+1}} & \text { in } M \\
\frac{\partial u}{\partial \nu}=0 & \text { on } \partial M .
\end{array}\right.
$$

We can show that the above equation has a unique solution $u(x) \equiv 0$. In fact, it is obvious that $u \equiv 0$ is a solution. Now we are going to prove its uniqueness.

At the maximum point $x_{0}$ of $u$, no matter if $x_{0}$ is an interior or a boundary point, we always have that $\left.\nabla u\right|_{x_{0}}=0$ and $\left.\nabla^{2} u\right|_{x_{0}}$ is nonpositive-definite. In fact if $x_{0}$ is an interior point, it is clear; if $x_{0}$ is a boundary point, we have $\left.\frac{\partial u}{\partial \nu}\right|_{\partial M}=0$ by equation (3.1) and $\left.\frac{\partial u}{\partial x^{\alpha}}\right|_{x_{0}}=0$, where $\left\{x^{\alpha}\right\}_{1 \leq \alpha \leq n-1}$ are local coordinates on the boundary $\partial M$ around $x_{0}$. Therefore $\left.\nabla^{2} u\right|_{x_{0}}$ is nonpositive-definite. Now at $x_{0}$ we have

$$
\begin{aligned}
\operatorname{vol}\left(M_{g}\right)^{\frac{2}{n+1}} & =\varsigma \cdot n \varrho=\varsigma F\left(\lambda\left(g^{-1} \cdot g\right)\right) \\
& \geq F\left(\lambda\left(g^{-1}\left[\varsigma g+\nabla^{2} u+d u \otimes d u-\frac{1}{2}|\nabla u|^{2} g\right]\right)\right) \\
& =\left(\int e^{-(n+1) u}\right)^{\frac{2}{n+1}}
\end{aligned}
$$

Similarly, at the minimum point of $u$, we can get $\varsigma \cdot n \varrho \leq\left(\int e^{-(n+1) u}\right)^{\frac{2}{n+1}}$. As a result, we have $\operatorname{vol}\left(M_{g}\right)^{\frac{2}{n+1}}=\varsigma \cdot n \varrho=\left(\int e^{-(n+1) u}\right)^{\frac{2}{n+1}}$.

By $\left(C_{6}\right)$, we know that $F \leq \varrho \sigma_{1}$. Hence,

$$
\begin{aligned}
\varsigma \cdot n \varrho & =F\left(\lambda\left(g^{-1}\left[\varsigma g+\nabla^{2} u+d u \otimes d u-\frac{1}{2}|\nabla u|^{2} g\right]\right)\right) \\
& \leq \varrho \sigma_{1}\left(\lambda\left(g^{-1}\left[\varsigma g+\nabla^{2} u+d u \otimes d u-\frac{1}{2}|\nabla u|^{2} g\right]\right)\right) \\
& =\varrho\left(n \varsigma+\triangle u+\left(1-\frac{n}{2}\right)|\nabla u|^{2}\right) .
\end{aligned}
$$

Then

$$
\left(\frac{n}{2}-1\right) \int_{M}|\nabla u|^{2} \leq \int_{M} \triangle u=\int_{\partial M} \frac{\partial u}{\partial \nu}=0,
$$

and $u \equiv$ const. $=0$.

Thus the operator

$$
\begin{aligned}
\Psi_{t}[u]= & F\left(\lambda\left(g^{-1}\left[\varsigma(1-\psi(t)) g+\psi(t) A_{g}+\nabla^{2} u+d u \otimes d u-\frac{1}{2}|\nabla u|^{2} g\right]\right)\right) \\
& -\psi(t) f(x) e^{-2 u}-(1-t)\left(\int e^{-(n+1) u}\right)^{\frac{2}{n+1}}
\end{aligned}
$$

satisfies the Leray-Schauder degree $\operatorname{deg}\left(\Psi_{0}, \mathcal{O}_{0}, 0\right) \neq 0$ at $t=0$, where the LeraySchauder degree is defined by [11] (see [2] for the boundary case) and $\mathcal{O}_{0}$ is a neighborhood of the zero solution in $\left\{u \in C^{4, \alpha}(M): \frac{\partial u}{\partial \nu}=0\right.$ on $\left.\partial M\right\}$. Thus, whereas we obtain the homotopy-invariance of the degree, we can derive that the Leray-Schauder degree is nonzero at $t=1$. This shows that equation (2.2) is solvable.

The $C^{1}$ and $C^{2}$ estimates of the solutions to (3.1) have been proved in 9 . We may obtain

Lemma 3.1. For any fixed $0<\delta<1$, there is a constant $C=C(\delta, n, g, f)$ such that any solution of (3.1) with $t \in[0,1-\delta]$ satisfies $\|u\|_{C^{4, \alpha}} \leq C$. 
So without loss of generality, we may assume that $u_{t_{i}}$ tends to $-\infty$ at $t_{i} \rightarrow 1$, where $u_{t_{i}}$ is the solution of (3.1) at $t=t_{i}$, which will be denoted by $u_{i}$ in what follows. Thus equation (3.1) turns out to be

$$
\left\{\begin{array}{lr}
F\left(\lambda\left(g^{-1}\left[A_{g}+\nabla^{2} u+d u \otimes d u-\frac{1}{2}|\nabla u|^{2} g\right]\right)\right) & \text { in } M \\
=(1-t) o+f(x) e^{-2 u} & \text { on } \partial M \\
\frac{\partial u}{\partial \nu}=0 & \text { on }
\end{array}\right.
$$

where $u$ is assumed to be admissible and $o \geq 0$ is a constant.

Furthermore, we can get a more exact estimate on the geodesic ball $B(x, r)=$ $\{y \in M \mid \operatorname{dist}(x, y)<r\}$ :

Lemma $3.2(9])$. Let $u \in C^{4}(M)$ be a $k$-admissible solution of (3.1) in $B(x, r)$ and $0 \leq r<1$. Then there is a constant $C=C(n, g, f)$ such that

$$
\left(\left|\nabla^{2} u\right|+|\nabla u|^{2}\right)\left(x^{\prime}\right) \leq C\left(r^{-2}+\exp \left(-2 \inf _{B(x, 2 \sqrt{10} r)} u\right)\right)
$$

for all $x^{\prime} \in B(x, r)$.

\section{Proof of Theorem 1.1}

We call $\left\{u_{k}\right\}$ the blow-up sequence and $\bar{x} \in M$ the blow-up point if $u_{k}\left(x_{0, k}\right) \rightarrow$ $-\infty$ as $x_{0, k} \rightarrow \bar{x}$, where $\left\{x_{0, k}\right\} \subset M$. Now let $\left\{u_{k}\right\}$ be a blow-up solution of (3.2) with the blow-up point $\bar{x}$.

First of all, we would like to prove that $\bar{x}$ can be approximated by local minimum points of $u_{k}$. Let $v_{k}=e^{-(n-2) / 2 u_{k}}$, denote $v_{k}\left(x_{0, k}\right)^{\frac{1}{n-2}}$ by $R_{0, k}$ and $\frac{1}{1-e^{-1 / 2}}$ by $A_{0}$.

Lemma 4.1. In each geodesic ball $B\left(x_{0, k}, A_{0} R_{0, k}^{-1}\right) \subset M$ we may find a local maximum point of $v_{k}$, denoted by $x_{k}$. Furthermore,

$$
v_{k}\left(x_{k}\right)=\sup _{B\left(x_{k}, v_{k}\left(x_{k}\right)^{-\frac{1}{n-2}}\right)} v_{k} .
$$

Proof. Let $e^{u_{k}\left(x_{0, k}\right)}=\varepsilon_{0, k}$. We define a mapping:

$$
\begin{aligned}
\mathcal{U}_{0, k}: \mathcal{B}\left(0, \varepsilon_{0, k}{ }^{-1 / 2}\right) \subset T_{x_{0, k}}(M) & \rightarrow B\left(x_{0, k}, \varepsilon_{0, k}{ }^{1 / 2}\right), \\
y & \longmapsto \exp _{x_{0, k}}\left(\varepsilon_{0, k} y\right),
\end{aligned}
$$

where the metric on the tangent space is $\check{g}_{k}=\varepsilon_{0, k}^{-2} \mathcal{U}_{0, k}^{*} g$ and $\mathcal{B}\left(0, \varepsilon_{0, k}{ }^{-1 / 2}\right)$ is a geodesic ball. Moreover, consider a sequence of functions $\mu_{0, k}(y)=u_{k}\left(\mathcal{U}_{0, k}(y)\right)-$ $\log \varepsilon_{0, k}$. We may derive an equation that $\mu_{0, k}(y)$ satisfies. In fact, we have

$$
\left\{\begin{array}{l}
F\left(\lambda\left(\check{g}_{k}^{-1}\left[A_{\check{g}_{k}}+\nabla^{2} \mu_{0, k}+d \mu_{0, k} \otimes d \mu_{0, k}-\frac{1}{2}\left|\nabla \mu_{0, k}\right|^{2} \check{g}_{k}\right]\right)\right) \\
=\varepsilon_{0, k}^{2}(1-t) o+f\left(\mathcal{U}_{0, k}(y)\right) e^{-2 \mu_{0, k}} \quad \text { in } \mathcal{B}\left(0, \varepsilon_{0, k}^{-1 / 2}\right), \\
\frac{\partial \mu_{0, k}}{\partial x^{n}}=0 \text { on } \mathcal{B}\left(0, \varepsilon_{0, k}{ }^{-1 / 2}\right) \cap\left\{x^{n}=0\right\}
\end{array}\right.
$$

where $\mu_{0, k}$ is admissible and $o$ is a nonnegative constant.

Let us begin with the easy case, $u_{k}(x) \geq u_{k}\left(x_{0, k}\right)-1$ in $B\left(x_{0, k}, \varepsilon_{0, k}{ }^{1 / 2}\right)$. In this case, $0 \leq e^{-\frac{n-2}{2} \mu_{0, k}} \leq e^{\frac{n-2}{2}}$ in $\mathcal{B}\left(0, \varepsilon_{0, k}-1 / 2\right)$. Hence, $\mu_{0, k}$ converges in $C^{3}$ to $\mu_{\infty}$ with $0 \leq e^{-\frac{n-2}{2} \mu_{\infty}} \leq e^{\frac{n-2}{2}}$ on $\mathbb{R}^{n}$, and the limit function $\mu_{\infty}$ satisfies

$$
\left.F\left(\lambda\left(\delta^{-1}\left[\nabla^{2} u+d u \otimes d u-\frac{1}{2}|\nabla u|^{2} \delta\right)\right]\right)\right)=f(\bar{x}) e^{-2 u} .
$$


Then by the Liouville Theorem [12, we know that 0 is the locally minimum point of $\mu_{0, k}$. Rescaling back, we see that $x_{0, k}$ is the locally minimum point of $u_{k}$ in $B\left(x_{0, k}, \varepsilon_{0, k}{ }^{1 / 2}\right)$.

The alternative case is that there exists $x_{1, k} \in B\left(x_{0, k}, \varepsilon_{0, k}{ }^{1 / 2}\right)$ such that $u_{k}\left(x_{1, k}\right)$ $<u_{k}\left(x_{0, k}\right)-1$. Then we may consider the lower bound of $u_{k}$ in $B\left(x_{1, k}, \varepsilon_{1, k}{ }^{1 / 2}\right)$, where $\varepsilon_{1, k}=e^{u_{k}\left(x_{1, k}\right)}<e^{-1} \varepsilon_{0, k}$. If $u_{k} \geq u_{k}\left(x_{1, k}\right)-1$ in $B\left(x_{1, k}, \varepsilon_{1, k}^{1 / 2}\right)$, then $\mu_{1, k}(y)=u_{k}\left(\mathcal{U}_{1, k}(y)\right)-\log \varepsilon_{1, k}>-1$, where

$$
\mathcal{U}_{1, k}: y \rightarrow \exp _{x_{1, k}}\left(\varepsilon_{1, k} y\right)
$$

and $x_{1, k}$ is a locally minimum point of $u_{k}$.

Otherwise, we may repeat the previous proceedings with $u_{k}\left(x_{j, k}\right)<u_{k}\left(x_{j-1, k}\right)-$ $1\left(x_{j, k} \in B\left(x_{j-1, k}, \varepsilon_{j-1, k}^{1 / 2}\right)\right), \quad \varepsilon_{j, k}=e^{u_{k}\left(x_{j, k}\right)}<e^{-1} \varepsilon_{j-1, k}$ and $\mu_{j, k}(y)$ $=u_{k}\left(\mathcal{U}_{j, k}(y)\right)-\log \varepsilon_{j, k}$, where

$$
\mathcal{U}_{j, k}: y \rightarrow \exp _{x_{j, k}}\left(\varepsilon_{j, k} y\right)
$$

For any given $k$, as $u_{k} \in C^{\infty}(M)$, there exists $j(k) \in \mathbb{N}, j(k)<\infty$ such that $u_{k}\left(x_{j(k), k}\right)<u_{k}\left(x_{j(k)-1, k}\right)-1$ and $u_{k} \geq u_{k}\left(x_{j(k), k}\right)-1$ in $B\left(x_{j(k), k}, \varepsilon_{j(k), k}{ }^{1 / 2}\right)$. Hence, we can find a locally minimum point of the $u_{k}$ in $B\left(x_{j(k), k}, \varepsilon_{j(k), k}{ }^{1 / 2}\right) \subset$ $B\left(x_{0, k}, A_{0} \varepsilon_{0, k}{ }^{1 / 2}\right)$. This completes the proof (see Lemma 3.2 in [15] for more details).

Now we consider the rescaled sequence $w_{k}=u_{k}-\sup _{M} u_{k}$. Suppose $x_{k}^{0}$ is the maximum point of $u_{k}$. Since $e^{-2 \sup u_{k}} f\left(x_{k}^{0}\right)=e^{-2 u_{k}\left(x_{k}\right)} f\left(x_{k}^{0}\right) \leq C\left(\triangle u_{k}+\right.$ $\left.A_{g}\right)\left(x_{k}^{0}\right) \leq C$, then $\bar{x}=\lim x_{k}$ is the blow-up point with respect to $w_{k}$ as well. It is obvious that $w_{k}$ satisfies the equation

$$
\left\{\begin{array}{lr}
F\left(\lambda\left(g^{-1}\left[A_{g}+\nabla^{2} w_{k}+d w_{k} \otimes d w_{k}-\frac{1}{2}\left|\nabla w_{k}\right|^{2} g\right]\right)\right) & \\
=(1-t) o+f(x) e^{-2 \sup _{M} u_{k}} e^{-2 w_{k}} & \text { in } M \\
\frac{\partial w_{k}}{\partial \nu}=0 & \text { on } \partial M
\end{array}\right.
$$

where $w_{k}$ is admissible and $o \geq 0$ is a constant.

By virtue of Lemma 4.1, we may assume that $\bar{x}=\lim x_{k}$, where $\left\{x_{k}\right\}$ are locally minimum points of $u_{k}$. Hence $\left\{x_{k}\right\}$ are also locally minimum points of $w_{k}$ and

$$
w_{k}\left(x_{k}\right)=\inf _{B\left(x_{k}, e^{\frac{1}{2} w_{k}\left(x_{k}\right)}\right)} w_{k} .
$$

Note that $F$ satisfies $\left(C_{1}\right)-\left(C_{6}\right)$ and the $w_{k}$ are $\Gamma$ admissible, where $\Gamma \subset \Sigma_{\frac{1}{n-2}}$. Hence $w_{k}$ are subharmonic and satisfy

$$
W+\frac{1}{n-2} \sigma_{1}(W) g \geq 0,
$$

where $W=\nabla^{2} w_{k}+d w_{k} \otimes d w_{k}-\frac{1}{2}\left|\nabla w_{k}\right|^{2} g+A_{g}$. We need the idea of the minimal radial functions of $w$ in $B_{R}\left(x_{0}\right)$ ([15]):

$$
\widehat{w}(x)=\sup \left\{w(y): y \in \partial B_{r}\left(x_{0}\right), r=d\left(x, x_{0}\right) \leq R\right\},
$$

and we denote $\nabla^{2} \widehat{w}+d \widehat{w} \otimes d \widehat{w}-\frac{1}{2}|\nabla \widehat{w}|^{2} g+A_{g}$ by $\widehat{W}$. Now we are ready to prove the following. 
Propostion 4.2. Let $u_{j}$ be a blow-up sequence of solutions to (3.2). Then $w_{j}=$ $u_{j}-\sup _{M} u_{j}$ converges in $w^{1, p}$ (for any $1<p<\frac{n}{n-1}$ ) to an admissible function w. Moreover, if $\bar{x}$ is a blow-up point of $w$, then near $\bar{x}$,

$$
w(x)=2 \log d(x, \bar{x})+o(1)
$$

where $d(x, \bar{x})$ denotes the geodesic distance from $x$ to $\bar{x}$ with respect to the metric g. Furthermore, each blow-up point is isolated.

Proof. Since a similar proposition on a manifold without boundary has appeared in [15], we only focus on the differences.

Step 1. We may get admissible solutions on the doubled manifold. Glue two copies of $(M, g)$ together along the totally geodesic boundary and denote the doubled manifold by $\dot{M}$. With the given smooth Riemannian metric $g$ on $M$, there is a standard metric $\check{g}$ on $\check{M}$ induced from $g$. When $\partial M$ is totally geodesic in $(M, g)$, $\check{g}$ is $C^{2,1}$ on $\check{M}$ (see [3] ).

We can extend $w_{k}$ to a $C^{2}(\check{M})$ function $\check{w}_{k}$ as follows: Near the boundary we take Fermi coordinates, where $\breve{w}_{k}$ is then defined as

$$
\check{w}_{k}\left(x_{1}, \cdots, x_{n}\right)= \begin{cases}w_{k}\left(x_{1}, \cdots, x_{n}\right), & x_{n} \geq 0 \\ w_{k}\left(x_{1}, \cdots,-x_{n}\right), & x_{n} \leq 0 .\end{cases}
$$

Since $\frac{\partial w_{k}}{\partial \nu}=0$, it is easy to verify by definition that $\check{w}_{k} \in C^{2}(\check{M})$. As a matter of fact,

$$
\begin{gathered}
\lim _{x_{n} \rightarrow 0^{+}} \frac{\partial \check{w}_{k}}{\partial x^{n}}\left(x_{1}, \cdots, x_{n}\right)=\frac{\partial w_{k}}{\partial x^{n}}\left(x_{1}, \cdots, x_{n-1}, 0\right) \\
=0=-\frac{\partial w_{k}}{\partial x^{n}}\left(x_{1}, \cdots, x_{n-1}, 0\right)=\lim _{x_{n} \rightarrow 0^{-}} \frac{\partial \check{w}_{k}}{\partial x^{n}}\left(x_{1}, \cdots, x_{n}\right)
\end{gathered}
$$

and

$$
\lim _{x_{n} \rightarrow 0^{+}} \frac{\partial^{2} \check{w}_{k}}{\partial\left(x^{n}\right)^{2}}\left(x_{1}, \cdots, x_{n}\right)=\lim _{x_{n} \rightarrow 0^{-}} \frac{\partial^{2} \check{w}_{k}}{\partial\left(x^{n}\right)^{2}}\left(x_{1}, \cdots, x_{n}\right) .
$$

Thus from the admissible property of $w_{k}$ we know that $\check{w}_{k}$ is also admissible and satisfies (4.1).

Step 2. We can find convergent "minimal radial functions" on the doubled manifold. Inequality (4.1) says that $\check{w}_{k}$ is subharmonic. From Corollary 2.1 in [15, $\left\{\check{w}_{k}\right\}$ converges to a subharmonic function $\check{w}$ in $W^{1, p}$ (for any $1<p<\frac{n}{n-1}$ ). By Corollary 2.2 in [15, the corresponding minimal radial functions $\widehat{\breve{w}}_{k}$ also converge to $\widehat{\widetilde{w}}$. Noting that the minimal radial functions depend only on the distance to the center, by Corollary 2.1 and Corollary 2.2 in [15], we may obtain

$$
\widehat{\widetilde{w}}(r)=\lim _{k \rightarrow \infty} \widehat{\widetilde{w}}_{k}(r),
$$

where

$$
\widehat{\widetilde{w}}_{k}(r)=\sup \left\{\check{w}_{k}(y): y \in \partial B_{r}\left(x_{k}\right)\right\}
$$

and

$$
\widehat{\widetilde{w}}(r)=\sup \left\{\check{w}(y): y \in \partial B_{r}(\bar{x})\right\} .
$$

On the one hand, based on (4.3) and (4.1), we can get the following estimates:

$$
\widehat{\widetilde{w}}(x) \leq 2 \log d(x, \bar{x})+C .
$$


In fact, we may assume that $\widehat{\widetilde{w}}_{k}(r)=\check{w}_{k}\left(x_{r}\right), x_{r}=(0, \cdots, 0, r)$, and $\left|A_{g}\right| \leq C r / 2$. The $\widehat{\widetilde{w}}_{k}$ are still admissible and satisfy inequality (4.1). Thus

$$
\begin{aligned}
0 \leq & \left((n-2) \widehat{W}_{n n}+\Sigma_{i} \widehat{W}_{i i}\right)\left(x_{r}\right) \\
\leq & (n-1)\left(\widehat{\breve{w}}_{k}^{\prime \prime}+\left(\widehat{\widetilde{w}}_{k}^{\prime}\right)^{2}-\frac{g_{n n}}{2}\left(\widehat{\widetilde{w}}_{k}^{\prime}\right)^{2}+C r / 2\right) \\
& +\sum_{i=1}^{n-1}\left(\left(\frac{1}{r}+C\right) \widehat{\widetilde{w}}_{k}^{\prime}-\frac{g_{i i}}{2}\left(\widehat{\widetilde{w}}_{k}^{\prime}\right)^{2}+C r / 2\right) \\
\leq & (n-1)\left(\widehat{\widetilde{w}}_{k}^{\prime \prime}+\frac{1}{r} \widehat{\widetilde{w}}_{k}^{\prime}+C\left(\widehat{\widetilde{w}}_{k}^{\prime}+r\right)\right),
\end{aligned}
$$

where the last inequality comes from $\sum_{i} g_{i i} \geq n$. Hence,

$$
\left(\log \left(r \widehat{\widetilde{w}}_{k}^{\prime}+r^{2}\right)\right)^{\prime}+C \geq 0 .
$$

By taking a limit we get (4.4).

On the other hand, let $\widehat{\widetilde{v}}_{k}=e^{-(n-2) / 2 \widehat{\widetilde{w}}_{k}}$. From $\triangle \widehat{\widetilde{v}}_{k} \leq C \widehat{\tilde{v}}_{k} r$, we get

$$
\left[r^{n-1} \widehat{\widetilde{v}}_{k}^{\prime}\right]^{\prime} \leq C r^{n} \widehat{\widetilde{v}}_{k}
$$

Thus, by a direct calculation, we know that

$$
\widehat{\breve{w}}(x) \geq 2 \log d(x, \bar{x})+o(1) .
$$

Therefore

$$
\widehat{\widetilde{w}}(x)=2 \log d(x, \bar{x})+o(1) .
$$

Then the comparison principle helps us to deduce (4.2) from (4.5). Roughly speaking, since $\check{w}$ equals $\widehat{\widetilde{w}}$ at some points, the comparison principle implies that they are equal everywhere. That is,

$$
\check{w}(x)=2 \log d(x, \bar{x})+o(1) .
$$

(For more details, one may consult section 3 of [15].)

Proof of Theorem 1.1. As the proof of Proposition 4.2, we glue two copies of $(M, g)$ together. Denote the doubled manifold and functions by a "check" (e.g. $\check{M}, \check{w}$ ). Since the Ricci curvature Ric $_{e^{-2 \tilde{w}} g}$ is still nonnegative, by (4.5) and the Volume Comparison Theorem, there is at most one end away from the blow-up points. The metric $e^{-2 \check{w}} g$ is in fact a Euclidean one (see section 7 of [6] for details); namely, $(M, g)$ is conformally equivalent to the unit half sphere, which contradicts the assumption in Theorem 1.1. Therefore there is a unform $L^{\infty}$ bound for solutions. So the set of solutions is compact. This completes the proof of Theorem 1.1.

\section{ACKNOWLEDGEMENT}

The first author would like to thank her advisor, Professor Kefeng Liu, for his support and encouragement. 


\section{REFERENCES}

1. S. Chen, Conformal deformation on manifolds with boundary, Geom. Funct. Anal., 19 (2009), no. 4, 1029-1064. MR2570314

2. S. Chen, Boundary value problems for some fully nonlinear elliptic equations, Calc. Var. Partial Differential Equations, 30 (2007), no. 1, 1-15. MR2333094(2008e:35061)

3. José F. Escobar, The Yamabe problem on manifolds with boundary, J. Differential Geom, 35 (1992), no. 1, 21-84. MR 1152225 (93b:53030)

4. A. Gray and L. Vanhecke, The volumes of tubes about curves in a Riemannian manifold, Proc. London Math. Soc., 44 (1982), 215-243. MR647431 (83h:53031)

5. P.F. Guan and G.F. Wang, Conformal deformations of the smallest eigenvalue of the Ricci tensor, American Journal of Mathematics, 129 (2007), no. 2, 499-526. MR2306044 (2007m:53033)

6. M. Gursky and J. Viaclovsky, Prescribing symmetric functions of the eigenvalues of the Ricci tensor, Ann. of Math. (2) 166 (2007), 475-531. MR2373147(2008k:53068)

7. M. Gursky and J. Viaclovsky, Volume comparison and the $\sigma_{k}$-Yamabe problem, Adv. Math., 187 (2004), no. 2, 447-487. MR2078344 (2005f:53051)

8. M. Gursky and J. Viaclovsky, Fully nonlinear equations on Riemannian manifolds with negative curvature, Indiana Univ. Math. J., 52 (2003), 399-419. MR1976082 (2004a:53039)

9. Y. He and W. M. Sheng, On existence of the prescribing $k$-curvature problem on manifolds with boundary, preprint.

10. Y. He and W. M. Sheng, Local estimates for elliptic equations arising in conformal geometry, preprint.

11. Y.Y. Li, Degree theory for second order nonlinear elliptic operators and its applications, Comm. Partial Differential Equations, 14 (1989), no. 11, 1541-1578. MR.1026774 (90i:58022)

12. A. Li and Y.Y. Li, On some conformally invariant fully nonlinear equations, part II: Liouville, Harnack, and Yamabe, Acta Math., 195 (2005), 117-154. MR2233687(2007d:53053)

13. J.Y. Li and W.M. Sheng, Deforming metrics with negative curvature by a fully nonlinear flow, Calc. Var. Partial Differential Equations, 23 (2005), 33-50. MR2133660 (2005m:53121)

14. W.M. Sheng, N.S. Trudinger and X-J. Wang, The Yamabe problem for higher order curvatures, J. Diff. Geom., 77 (2007), 515-553. MR2362323 (2008i:53048)

15. N.S. Trudinger and X-J. Wang, The intermediate case of the Yamabe problem for higher order curvatures, International Mathematics Research Notices (2010) 2010 (13), 2437-2458.

16. X.-J. Wang, A class of fully nonlinear elliptic equations and related functionals, Indiana Univ. Math. J., 43 (1994), 25-54. MR1275451 (95f:35089)

Centre for Mathematical Sciences, Zhejiang University, Hangzhou 310027, People's Republic of China

E-mail address: helenaig@zju.edu.cn

Department of Mathematics, Zhejiang University, Hangzhou 310027, People's RepubLIC OF CHINA

E-mail address: weimins@zju.edu.cn 